\title{
RNA-sequencing reveals altered skeletal muscle contraction, E3 ligases, autophagy, apoptosis, and chaperone expression in patients with critical illness myopathy
}

Monica Llano-Diez ${ }^{1 \dagger}$, Wen Fury ${ }^{2+}$, Haruka Okamoto ${ }^{2}$, Yu Bai ${ }^{2}$, Jesper Gromada ${ }^{2}$ and Lars Larsson ${ }^{1,3^{*}}$ (D)

\begin{abstract}
Background: Critical illness myopathy (CIM) is associated with severe skeletal muscle wasting and impaired function in intensive care unit (ICU) patients. The mechanisms underlying CIM remain incompletely understood. To elucidate the biological activities occurring at the transcriptional level in the skeletal muscle of ICU patients with CIM, the gene expression profiles, potential upstream regulators, and enrichment pathways were characterized using RNA sequencing (RNA-seq). We also compared the skeletal muscle gene signatures in ICU patients with CIM and genes perturbed by mechanical loading in one leg of the ICU patients, with an aim of reducing the loss of muscle function.
\end{abstract}

Methods: RNA-seq was used to assess gene expression changes in tibialis anterior skeletal muscle samples from seven critically ill, immobilized, and mechanically ventilated ICU patients with CIM and matched control subjects. We also examined skeletal muscle gene expression for both legs of six ICU patients with CIM, where one leg was mechanically loaded for $10 \mathrm{~h} /$ day for an average of 9 days.

Results: In total, 6257 of 17,221 detected genes were differentially expressed (84\% upregulated; $p<0.05$ and fold change $\geq 1.5$ ) in skeletal muscle from ICU patients with CIM when compared to control subjects. The differentially expressed genes were highly associated with gene changes identified in patients with myopathy, sepsis, long-term inactivity, polymyositis, tumor, and repeat exercise resistance. Upstream regulator analysis revealed that the CIM signature could be a result of the activation of MYOD1, p38 MAPK, or treatment with dexamethasone. Passive mechanical loading only reversed expression of $0.74 \%$ of the affected genes ( 46 of 6257 genes).

Conclusions: RNA-seq analysis revealed that the marked muscle atrophy and weakness observed in ICU patients with CIM were associated with the altered expression of genes involved in muscle contraction, newly identified E3 ligases, autophagy and calpain systems, apoptosis, and chaperone expression. In addition, MYOD1, p38 MAPK, and dexamethasone were identified as potential upstream regulators of skeletal muscle gene expression in ICU patients with CIM. Mechanical loading only marginally affected the skeletal muscle transcriptome profiling of ICU patients diagnosed with CIM.

Keywords: Critical illness myopathy, RNA sequencing, Skeletal muscle transcriptomics, Gene expression, Intensive care unit, Mechanical loading, Muscle atrophy

\footnotetext{
* Correspondence: lars.larsson@ki.se

${ }^{\dagger}$ Monica Llano-Diez and Wen Fury contributed equally to this work.

${ }^{1}$ Department of Physiology and Pharmacology, Karolinska Institutet,

Bioclinicum, J8:30, SE-171 77 Stockholm, Sweden

${ }^{3}$ Department of Clinical Neuroscience, Karolinska Institutet and Karolinska

Hospital, Stockholm, Sweden

Full list of author information is available at the end of the article
}

(c) The Author(s). 2019 Open Access This article is distributed under the terms of the Creative Commons Attribution 4.0 International License (http://creativecommons.org/licenses/by/4.0/), which permits unrestricted use, distribution, and

reproduction in any medium, provided you give appropriate credit to the original author(s) and the source, provide a link to the Creative Commons license, and indicate if changes were made. The Creative Commons Public Domain Dedication waiver (http://creativecommons.org/publicdomain/zero/1.0/) applies to the data made available in this article, unless otherwise stated. 


\section{Background}

The ability of the skeletal muscle to sense mechanical stimuli is important for regulation of gene expression and protein synthesis and enables the muscle to adapt to altered physiological demands. The process is referred to as tensegrity $[1,2]$. Conversely, loss of tensegrity due to mechanical silencing (absence of external strain related to weight-bearing and internal strain in the muscle fiber caused by myosin-actin activation) causes muscle wasting and weakness. This explains in part why critically ill, immobilized, and mechanically ventilated intensive care unit (ICU) patients develop severe muscle wasting and impaired muscle function $[3,4]$. The most frequent primary myopathy causing muscle weakness and paralysis in the ICU is called critical illness myopathy (CIM), which is found in up to $30 \%$ of the general ICU population and up to $100 \%$ in certain subgroups [5]. Moreover, CIM has been associated with delayed recovery, increased morbidity and financial costs, and impaired quality of life among survivors $[6,7]$. CIM is characterized by marked myosin loss, muscle atrophy affecting muscle fibers expressing type I and II myosin heavy chain (MyHC) isoforms, and reduced muscle membrane excitability $[5,8]$. The pathophysiology of CIM is complex and remains incompletely understood, but it involves activation of protein degradation pathways, transcriptional downregulation of myofibrillar proteins, decreased membrane excitability, mitochondrial dysfunction, and altered excitation-contraction coupling [9, 10]. Microarray technology has been used to elucidate skeletal muscle gene expression changes in critically ill and mechanically ventilated ICU patients and in animal models mimicking the ICU condition [11-13], but high-throughput RNA sequencing (RNA-seq) studies to identify differentially expressed genes (DEGs) and upstream regulators in ICU patients with CIM are still lacking. The aim of this study was to investigate gene expression changes in skeletal muscle from critically ill and mechanically ventilated ICU patients with CIM to understand the pathophysiological processes occurring in the skeletal muscle from CIM patients and to provide novel insight into the mechanisms underlying muscle weakness in ICU patients.

No specific and effective treatment is available for CIM. Supportive strategies such as early rehabilitation help improve patients' recovery and functional outcomes. There has been an interest in the study of the effects of passive movement on the skeletal muscle to preserve the architecture of the muscle fibers and prevent protein loss and function in mechanically ventilated and paralyzed critically ill ICU patients [14]. Our previous work showed that passive mechanical loading had positive effects on muscle fiber function in both experimental and clinical studies $[15,16]$, but the mechanisms underlying this finding remain incompletely understood. Consequently, an additional aim of this study was to explore if preservation of muscle function by mechanical loading for $10 \mathrm{~h}$ a day for an average of 9 days on one leg of ICU patients affected by CIM reduced the disease gene signature when compared to the unloaded leg from the same patient.

\section{Methods}

\section{Patients and control subjects}

Percutaneous conchotome muscle biopsies from the tibialis anterior (TA) muscle were used for RNA-seq from seven mechanically ventilated ICU patients (five women aged 4180 years and two men aged 55 and 68 years) and six control subjects (five women aged 68-82 years and one man aged 72 years). The majority of the patients were neuro-ICU patients $(n=4)$ who were immobilized after neurosurgery, cerebral lesions and infections. The other patients were mechanically ventilated and immobilized due to sepsis after a colon perforation, chronic obstructive pulmonary disease with pneumonia, and thrombocytopenia purpura with neurological and renal complications. All patients had been mechanically ventilated for longer than 2 weeks at the time of muscle biopsy and electrophysiological examination. All patients were diagnosed with CIM based on electrophysiological findings that reflected reduced compound muscle action potentials upon supramaximal stimulation of motor nerves, normal or subnormal motor nerve conduction velocities, and the presence of spontaneous electromyographic activity (typically positive sharp waves and fibrillation potentials) in some of the proximal and distal upper and lower extremity muscle investigated; additionally, patients displayed the hallmark of CIM, i.e., the preferential loss of the motor protein myosin when normalized to actin content $[10,17]$. Myosin to actin ratios were measured as previously described [15]. The average myosin to actin ratios in patients with CIM were $0.65 \pm 0.43$ (all patients had a myosin to actin ratio below 1.5). In a separate study, muscle biopsies were simultaneously obtained bilaterally from the TA in seven mechanically ventilated neuro-ICU patients, where one leg was subjected to mechanical loading for $2.5 \mathrm{~h}$, four times a day for $9 \pm 1$ days. The donor characteristics and the mechanical loading procedure have been described [15]. The muscle biopsies were obtained using the percutaneous conchotome method on the final day of the observation period. Each biopsy was dissected and treated as previously described [15, 18]. Written informed consent was obtained from patients' close relatives prior to the study. The study was approved by the Ethical Committee on Human Research at Karolinska Institutet, Stockholm and Uppsala University Hospital, Uppsala, Sweden.

\section{Transcriptome sequencing data generation}

The protocols for RNA extraction and sequencing library preparation were similar to those described earlier [19]. 
The RNA samples were treated with DNase prior to RNA-seq using the RNase-free DNase kit according to the manufacturer's instructions (Qiagen, Inc., Valencia, CA). An Advanced Analytical Fragment Analyzer was used for RNA quality analysis, and the RNA quality number $(\mathrm{RQN})$ is included in Additional file 1. Strand-specific RNA-seq libraries were prepared from 500-ng RNA using the KAPA stranded mRNA-Seq Kit (KAPA Biosystems, Wilmington, MA, USA). The libraries were amplified by 12-cycle PCR. Sequencing was performed on an Illumina HiSeq 2000 (Illumina) by multiplexed single-read run with 33 cycles. The resulting FASTQ files were analyzed via FastQC to ensure sufficient data quality. The reads were mapped to the human genome (hg19) using commercial software ArrayStudio (OmicSoft) with two mismatches allowed. The average reads sequenced was about 16.8 million per sample and the average reads aligned to transcripts was about 97.0\%. Among them, 83-90\% reads were uniquely mapped.

\section{Differential gene expression analysis}

Gene expression was quantified by the number of reads mapped to the sense-strand exons and converted to reads per kilobase per million (RPKM). Genes were flagged as detectable with an empirical minimum RPKM of 0.1. For comparison between two groups of samples, genes were eliminated if they were not detectable in the number of samples that is greater or equal to the smaller sample size of the two comparing groups. Fold changes were computed for the remaining genes as the ratio between the arithmetic mean RPKM values of the two groups. The statistical significance of the differential expression was assessed by Student's $t$ test. Genes with a fold change $\geq 1.5$ in either direction and with a $p<0.05$ were reported as significantly differentially expressed genes (DEGs). False discovery rate (FDR) was also calculated and provided, even though the values were not used as part of the threshold to obtain significant genes.

Gene Ontology (GO) biological process analysis was performed with the PANTHER Overrepresentation Test (released 2017205) in PANTHER ${ }^{\mathrm{TM}}$ version 13.1 (http://www. pantherdb.org/). The topmost significantly differentially regulated 1266 genes were used; these genes were ranked by absolute fold change after meeting the thresholds of $p<$ 0.05 and fold change $\geq 3.0$, as determined by Fisher's exact test with the FDR multiple test correction. In addition, some GO categorization was performed manually (independent of PANTHER) to improve the interpretative value of the data.

\section{Pathway enrichment analysis}

The resulting DEG signatures were compared with the canonical pathways in MSigDB. The statistical significance of the overlap between individual pathways with a given list of gene signatures was evaluated by NextBio Running Fisher test. Pathways were ranked by their significance, and the 15 top pathways were reported herein.

\section{Ingenuity upstream regulator and pathway analysis}

The topmost significantly perturbed skeletal muscle genes in CIM patients when compared to healthy control subjects ( $t$ test $p<0.05$ and fold change $>5$ in either direction) were subjected to Ingenuity Upstream Analysis (IPA, QIAGEN Redwood City, www.qiagen.com/ingenuity). The most significant upstream analysis results were reported based on $p<1.0 \mathrm{E}-4$ and activation $z$-score $>2$. Genes differentially expressed in the passively exercised leg and the nonloaded control legs ( $t$ test $p<0.05$ and fold change $>1.5$ in either direction) were also analyzed by Ingenuity Canonical Pathway analysis.

\section{Results}

Gene signature of skeletal muscle from ICU patients with CIM

We performed transcriptome profiling on the TA skeletal muscles isolated from seven critically ill patients who had been mechanically ventilated in the ICU for more than 2 weeks and six healthy control subjects. We detected 17,221 expressed genes when counting genes with RPKM over 0.1 in at least 6 out of the 13 samples. Figure 1a shows that the skeletal muscle samples from the ICU patients, and corresponding healthy control subjects were well separated into distinct clusters in principal component analysis (PCA) using all genes detected. When comparing the skeletal muscle gene signatures of the ICU patients with CIM to the healthy controls, we found 6257 DEGs $(p<0.05$ and fold change $\geq 1.5$ ) (Additional file 2). Most of the affected genes were upregulated ( $84 \%$ or 5237 genes), and only $16 \%$ (1020 genes) were downregulated. The heat map in Fig. $1 \mathrm{~b}$ shows very similar changes in the expression of the top 1266 differentially expressed genes $(p<0.05$ and fold difference $\geq 3$ ) among the ICU muscle samples when normalized against the mean of the healthy controls. Table 1 shows the 15 most significant results of the NextBio correlation analysis. The DEGs in the patients with CIM were highly associated with gene changes identified in patients with myopathy, sepsis, long-term inactivity, polymyositis, tumor, and repeated resistance exercise. Collectively, these data show that the expression of $36 \%$ of all detected genes was affected in TA skeletal muscle from patients with CIM and that the gene signatures associated highly with changes were identified in patients with diseases that either directly or indirectly affect skeletal muscle mass and function.

To obtain more detailed gene expression patterns, selected DEGs associated with GO biological process 
A

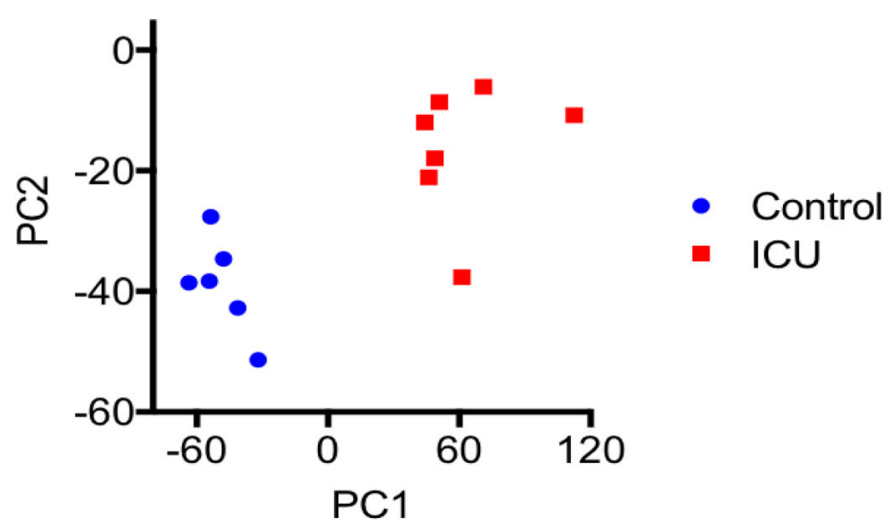

B

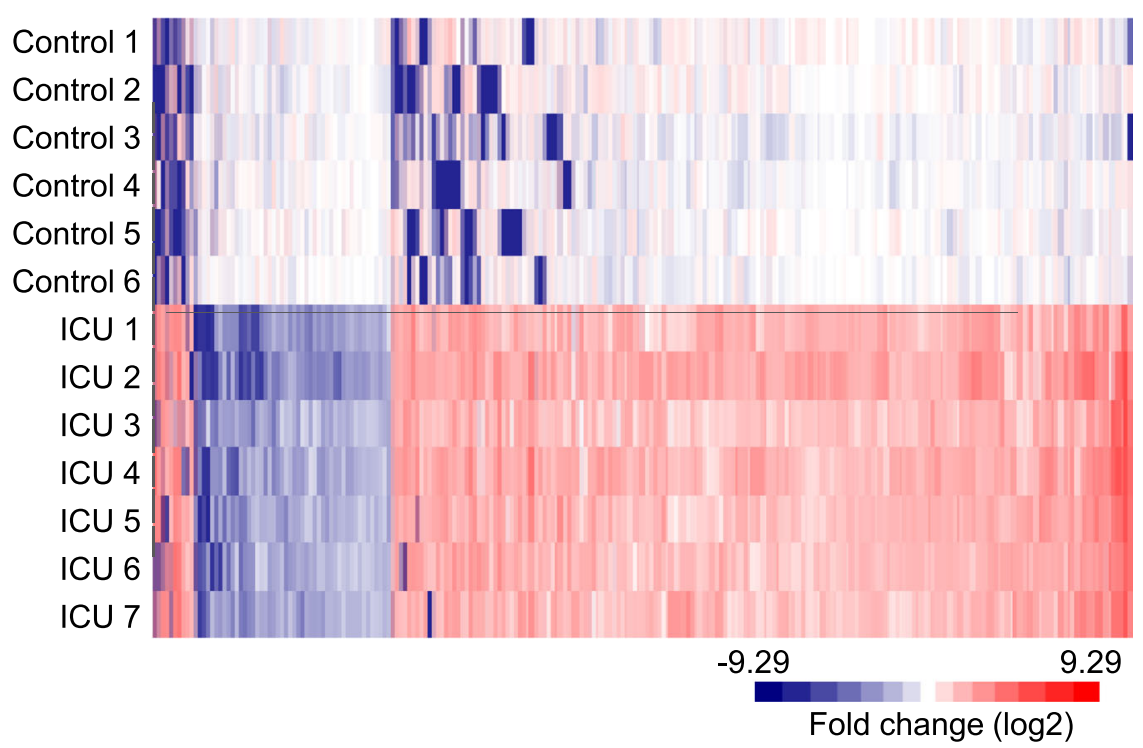

Fig. 1 PCA and heat map of DEGs of muscle samples from ICU patients and healthy controls. a PCA of TA skeletal muscle samples from healthy control subjects and ICU patients with CIM using all genes (RPKM log2 transformed and mean centered) b Heat map of top 1266 DEGs between ICU patients with CIM and healthy control muscle samples (independent group $t$ test $p<0.05$ and fold difference $\geq 3$ ). RPKM of each gene was normalized against the mean of healthy controls and followed by log2 transformation

categorization are discussed below (for fold change and $p$ values, please see Additional file 2).

\section{Muscle contraction and system process}

The software program PANTHER identified "muscle contraction" and "muscle system process" as the most significantly enriched GO terms (fold enrichment 2.89 and 3.05, FDR 3.65E-03 and 1.52E-04, respectively) for the top 1266 DEGs with fold change $\geq 3$ and $p<0.05$. Within this category and when focusing on skeletal muscle contraction, the gene encoding the $\beta$ /slow type I MyHC isoform (MYH7), the dominant isoform in TA muscle, was downregulated. Myosin light chains (MYL-1, 2 ,3, and MYLK2), actinin-3 (ACTN3), myotilin $(M Y O T)$, and structural sarcomeric proteins such as myomesin-2 (MYOM2) were also downregulated. The loss of myosin and myosin-associated proteins is a hallmark of CIM, and the results at the transcriptional level agree with clinical and experimental CIM studies $[4,12,15]$. Actin (ACTA1) expression was downregulated in patients with CIM, although the myosin to actin ratios decreased at the protein level. Myogenin (MYOG), myogenic factor (MYF6), calmodulin-1 (CALM1), dystrophia myotonica protein kinase (DMPK), and several subunits of acetylcholine receptor (CHRN-A1, B1, D, G) and sodium channel $(S C N-9 A, 5 A, 3 B)$ were highly upregulated in skeletal muscle of patients with CIM, whereas nitric oxide synthase 1 (NOS1) was downregulated.

Out of the top 1266 DEGs, myosin binding protein $\mathrm{H}$ $(M Y B P H)$, tropomodulin-1 (TMOD1), and some subunits of dihydropyridine-sensitive L-Type (CACN-B4, B1, G8) were upregulated, whereas, myosin binding protein $\mathrm{C} 1$ (MYBPC1), tropomyosin (TPM3), and troponin T1 
Table 1 NextBio correlation analysis of genes perturbed in the muscle of ICU patients. DEGs between ICU patients with CIM and healthy control muscle samples were used as input. The top 15 most significant results are listed

\begin{tabular}{|c|c|c|c|c|c|c|c|}
\hline \multirow[b]{2}{*}{ Project ID } & \multirow[b]{2}{*}{ Sample } & \multirow[b]{2}{*}{ Species } & \multicolumn{2}{|l|}{ Comparisons } & \multicolumn{3}{|c|}{ Correlations to this study } \\
\hline & & & Bioset 1 & Bioset 2 & $p$ value & Direction & $\begin{array}{l}\text { Number of } \\
\text { common genes }\end{array}$ \\
\hline GSE3307 & Skeletal muscle & Homo sapiens & $\begin{array}{l}\text { Acute quadriplegic } \\
\text { myopathy patients }\end{array}$ & Healthy subjects & $6.00 \mathrm{E}-87$ & + & 2607 \\
\hline GSE13205 & Vastus lateralis & Homo sapiens & $\begin{array}{l}\text { Septic patients with } \\
\text { organ failure }\end{array}$ & Healthy subjects & $1.00 \mathrm{E}-73$ & + & 687 \\
\hline GSE38680 & Biceps & Homo sapiens & $\begin{array}{l}\text { Infantile-onset } \\
\text { Pompe patients }\end{array}$ & Healthy subjects & 1.50E-68 & + & 2779 \\
\hline GSE24215 & Vastus lateralis & Homo sapiens & 9 days of inactivity & Baseline & $2.80 \mathrm{E}-54$ & + & 840 \\
\hline GSE26852 & Skeletal muscle & Homo sapiens & Polymyositis patients & Healthy subjects & $7.20 \mathrm{E}-51$ & + & 1148 \\
\hline GSE17679 & Skeletal muscle & Homo sapiens & $\begin{array}{l}\text { Ewing's sarcoma } \\
\text { recurrent tumor }\end{array}$ & Normal tissue & $8.10 \mathrm{E}-50$ & + & 3980 \\
\hline GSE17679 & Skeletal muscle & Homo sapiens & $\begin{array}{l}\text { Ewing's sarcoma } \\
\text { metastasis }\end{array}$ & Normal tissue & $5.40 \mathrm{E}-46$ & + & 4225 \\
\hline GSE17679 & Skeletal muscle & Homo sapiens & $\begin{array}{l}\text { Ewing's sarcoma } \\
\text { primary tumor }\end{array}$ & Normal tissue & $5.50 \mathrm{E}-46$ & + & 4451 \\
\hline GSE45426 & Vastus lateralis & Homo sapiens & $\begin{array}{l}3 \text { days of repeat } \\
\text { resistance exercise }\end{array}$ & Baseline & $6.60 \mathrm{E}-44$ & + & 2212 \\
\hline GSE17503 & Paravertebral muscle cells & Homo sapiens & Cultured & Biopsy without culture & $1.90 \mathrm{E}-42$ & + & 2045 \\
\hline GSE38012 & Vastus lateralis & Homo sapiens & $\begin{array}{l}\text { Subjects on long-term } \\
30 \% \text { calorie restricted diet }\end{array}$ & Subjects on Western diet & $1.10 \mathrm{E}-41$ & - & 3239 \\
\hline GSE14901 & Vastus lateralis & Homo sapiens & $\begin{array}{l}14 \text { days of limb } \\
\text { immobilization by cast }\end{array}$ & Baseline & $1.60 \mathrm{E}-41$ & + & 1274 \\
\hline GSE1551 & Muscle & Homo sapiens & Dermatomyositis patients & Healthy subjects & $8.90 \mathrm{E}-40$ & + & 1239 \\
\hline GSE4411 & Tibialis anterior muscles & Mus musculus & 3 days of denervation & No denervation & $9.60 \mathrm{E}-40$ & + & 1748 \\
\hline GSE26852 & Skeletal muscle & Homo sapiens & Dermatomyositis patients & Healthy subjects & 4.80E-39 & + & 1027 \\
\hline
\end{tabular}

(TNNI1) were downregulated. Moreover, genes that play a critical role in skeletal muscle development and regeneration were upregulated, such as the metalloprotease-14 (MMP14) and the transcription factor SOX6.

\section{Ubiquitin-proteasome system (UPS)}

Many genes involved in UPS, such as the proteasome family (PSM), ubiquitin-conjugating enzymes (UBEs), and E3 ligases, were altered in response to the ICU intervention. The two well-studied E3 ubiquitin ligases, MURF1/TRIM63 and ATROGIN-1/FBXO32, showed upregulation, but they did not pass the $p$ value cutoff. The recently discovered E3 ligases FBXO30/MUSA1 and TRIM32 were upregulated, whereas $F B X O 40$ expression was reduced.

\section{Autophagy-lysosome system (ALS)}

The cathepsins (CTSS, CTSB, CTSA, CTSD, CTSZ, and $L G M N)$, which are lysosomal proteases, were upregulated, despite the upregulation of several cathepsin inhibitors. The microtubule-associated protein 1 light chain 3 beta (MAP1LC3B), p62/SQSTM1, and the nutrient-deprivation autophagy factor-1 (NAF1) had increased expression
(MAP1LC3B did not pass the $\mathrm{p}$ value cutoff). The transcriptional upregulation of these genes is important during the activation of autophagy [20].

\section{Calpain system}

The expression of calpains, nonlysosomal calciumdependent proteases, changed in CIM patients. CAPN-1, 2, 10, and CAPNS1 were upregulated, while CAPN6, $C A P N 8$, and the muscle-specific isoform, $C A P N 3$, were downregulated.

\section{Protein folding and heat shock proteins/chaperones}

Proteins with chaperone functions are important for the control of protein folding and the reduction of misfolded protein aggregates. There was an upregulation of a large number of chaperones at the RNA level in patients with CIM; the key endoplasmic reticulum chaperones, calnexin $(C A N X)$ and calreticulin $(C A L R)$, tubulin-specific chaperones (TBCA and TBCC), members of the torsin family (TOR1A, TOR2A, and TOR $3 A$ ), and several members of the TCP-1 ring complex (TRiC, also called chaperonin containing TCP-1 [CCT]) were upregulated. The myosin chaperone $U N C-45 B$, regulating myosin folding, 
assembly, and function, was upregulated together with heat shock protein-90 (HSP90AA1, HSP90AB1, and HSP90AB4P). HSP70 (HSPA2) and HSP20 (HSPB6) expression was downregulated in ICU patients with CIM.

\section{Apoptosis}

The effector caspases, CASP4 and CASP6, which execute apoptosis were upregulated, as were other genes that regulate apoptosis, such as programmed cell death 6 (PDCD6), apoptosis antagonizing transcription factor $(A A T F)$, apoptotic chromatin condensation inducer 1 (ACIN1), apoptosis inducing factor mitochondria associated 1 (AIFM1), apoptotic peptidase activating factor 1 (APAF1), makorin ring finger protein 1 (MKRN1), BCL2 associated $\mathrm{X}$ apoptosis regulator $(B A X)$, heat shock protein beta1 (HSPB1), and the E3 ubiquitin ligase $R B X 1$. TP53 (p53) and its regulated genes, such as CDKN1A (p21), GADD45A, PIDD, BAX, and DDB2 (p48), were also upregulated. Apoptosis can also be induced through the activation of death receptors, including tumor necrosis factor (TNF family), which was altered in patients with CIM.

\section{mRNA translation}

The eIF2 initiation complex regulates mRNA translation. Several genes of this pathway, including EIF2 members (EIF2, EIF2AK4, EIF2B4, EIF2B2, EIF2S2), many EIF3, EIF4, and EIF5 complex members, and ribosomal subunits, were upregulated, among other genes involved in the pathway, which suggests an activation of muscle protein synthesis in patients with CIM.

\section{MYOD1, p38 MAPK, and dexamethasone were identified} as potential upstream regulators of skeletal muscle gene expression in ICU patients with CIM

Ingenuity upstream regulator analysis was employed to study 361 genes, and a set of top differentially regulated genes among the ICU muscle samples were identified by the following criteria: $p<0.05$ and fold change $>5$. The analysis examines the enrichment of the selected genes in known pathways downstream of a regulator and predicts the direction of activity change (activation $z$-score) for the upstream regulator in relation to what is expected from the literature. We applied cutoff criteria of $p<1.0 \mathrm{E}-4$ and the absolute value of activation $z$-score $>2$ for either direction. The analysis revealed that genes controlled by MYOD1, p38 MAPK, and dexamethasone were affected in the ICU patients with CIM. These regulators have established roles in skeletal muscle development and regeneration, as well as in response to changes in muscle size [21-24]. They had positive $z$-scores, indicating that activation of MYOD1, p38 MAPK, or the application of dexamethasone could cause gene expression changes similar to those observed in these patients (Fig. 2).

\section{Effects of passive mechanical loading on skeletal muscle} gene signature in ICU patients with CIM

Next, we obtained gene signatures from TA skeletal muscle samples from both legs of six critically ill and immobilized ICU patients who developed CIM; one leg (left) was subjected to passive mechanical loading for $10 \mathrm{~h}$ daily for an average of 9 days. In this analysis, we identified 16,594 genes, which were detected (RPKM > 0.1) in at least 6 of the 12 samples. PCA did not reveal a separation of the unloaded and loaded muscle samples (Fig. 3a). Passive mechanical loading of the left leg was previously reported to increase the specific force of single muscle fibers by $35 \%$, but it did not prevent the loss of myosin and muscle mass [25]. It was therefore surprising that we only detected 182 DEGs between muscle samples obtained from passive loaded left and unloaded right leg muscles of the patients with CIM (dependent group $t$ test $p<0.05$ and fold difference $\geq 1.5$ ) (Additional file 3). The heat map in Fig. 3b shows a fairly even expression of the 182 affected genes among patients when their expression was normalized to the unloaded control of the same patient. Ingenuity pathway analysis suggests that these genes were enriched in Phospholipase C Signaling $(p=5.48 \mathrm{E}-3)$ and Role of NFAT in Immune Response (9.01E-03). Only 68 of the 182 genes were in common with the 6257 affected genes in the patients with CIM (Fig. 4a). Of these 68 genes, 46 were regulated in the opposite direction, and 22 genes were regulated in the same direction (Additional file 4 and Fig. 4). Of the 46 genes regulated in the opposite direction, the expression of 38 genes was upregulated, and 8 were downregulated in the CIM state. The opposite effect of CIM state and mechanical loading on these overlap genes are statistically significant (Fisher's exact test, $p=0.046$ ), suggesting the possible positive effect of mechanical loading in these patients. Interestingly, we found that $\mathrm{MYH}$ C-IIX/D (MYH1) expression was sixfold higher in the muscle of ICU patients (Additional file 2), and it was further upregulated by mechanical loading (Fig. 5 and Additional file 3). Collectively, these and previous data show that mechanical loading of legs of ICU patients with CIM improves the specific force of single TA skeletal muscle fibers, despite a preservation of myosin loss and muscle wasting [15], and it reverses the expression of only 46 genes out of 6257 affected genes in the ICU patients with CIM.

We did not compare the samples from the two studies. The principal component analysis (PCA) suggested that samples from the two studies were separated. The lack of overlap between the CIM samples from the two studies could potentially be due to differences in procedures for sample acquisition, treatment, and handling. 


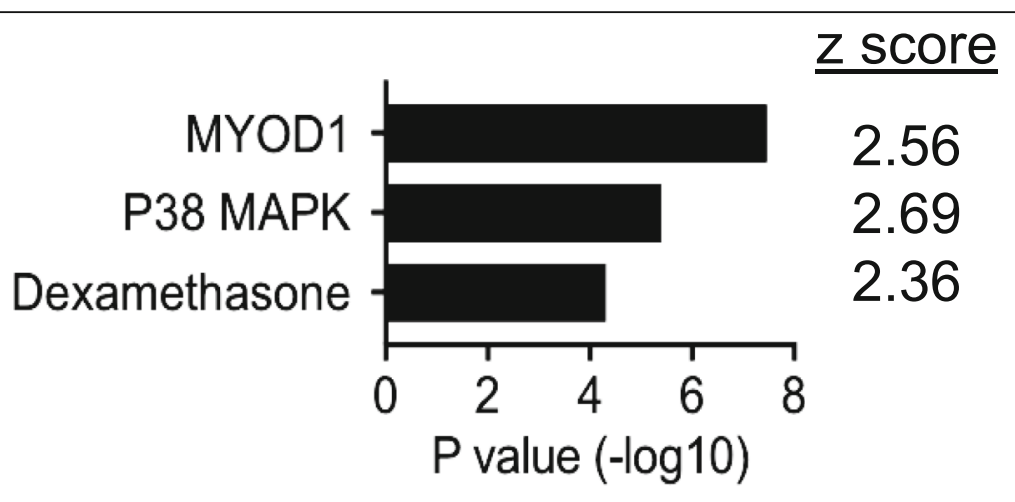

Fig. 2 Ingenuity upstream regulator analysis of top skeletal muscle genes perturbed in ICU patients with CIM. The most significantly perturbed genes in the ICU patients with CIM (361 genes, Student $t$ test $p<0.05$ and fold change $>5$ in either directions) were subjected to the analysis. Inclusion criteria were $p<1.0 \mathrm{E}-04$ and activation $z$-score $>2$
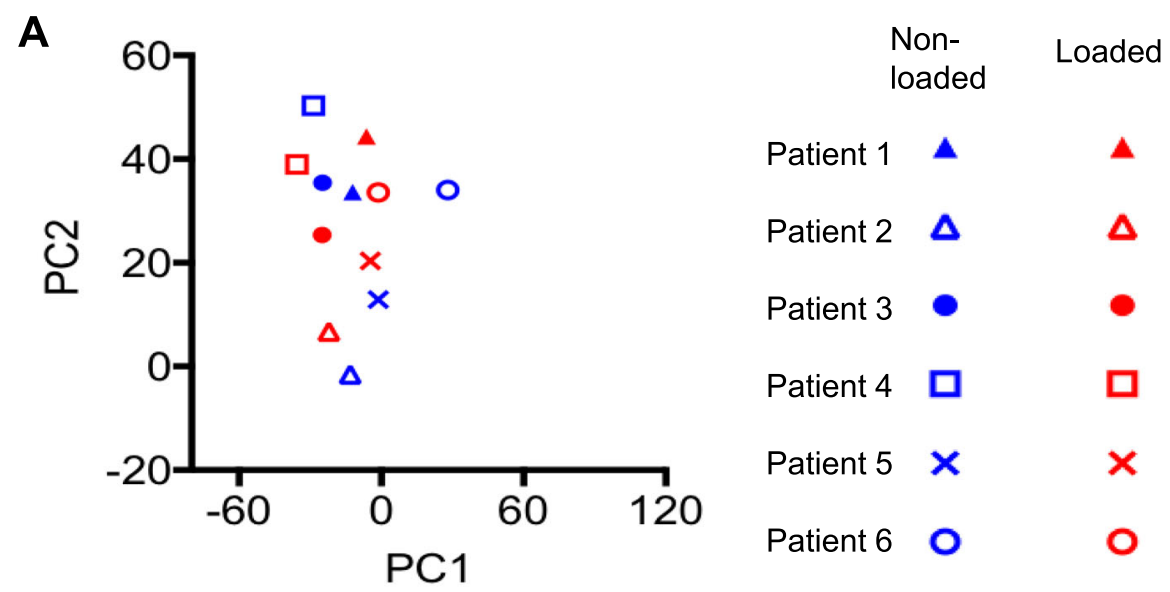

B

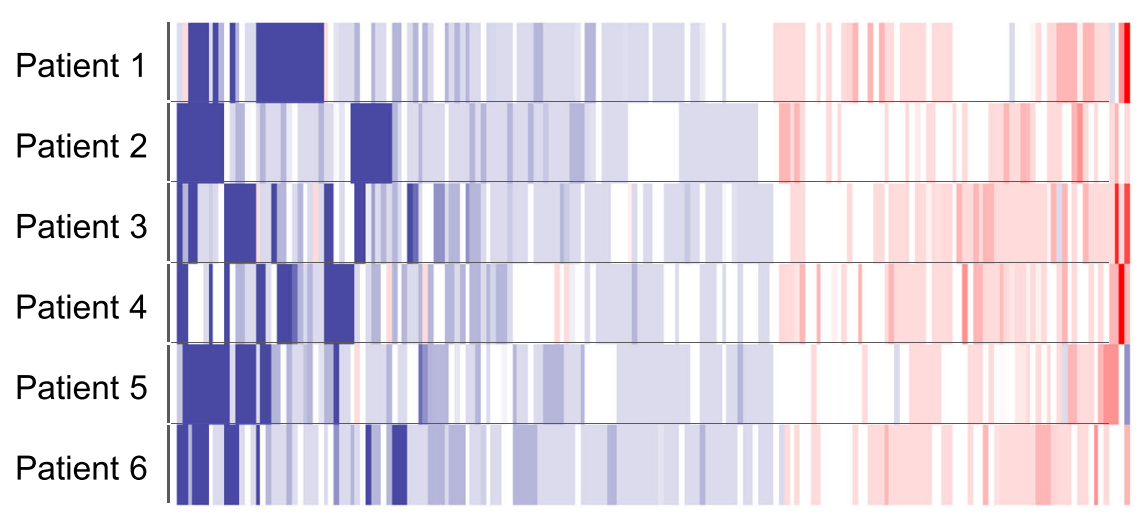

$-10.84$

10.84

Fold change ( $\log 2)$

Fig. 3 PCA and heat map of DEGs of CIM muscle samples with or without passive loading. a PCA of skeletal muscle samples from passively loaded left and nonloaded right legs of ICU patients with CIM using all genes detected (RPKM log2 transformed and mean centered) b Heat map of 182 DEGs between muscle samples obtained from passive loaded left and nonloaded right leg muscles of critically ill ICU patients with CIM (dependent group $t$ test $p<0.05$ and fold difference $\geq 1.5$ ). RPKM of each gene was normalized against the nonloaded control of the same patient and followed by $\log 2$ transformation 


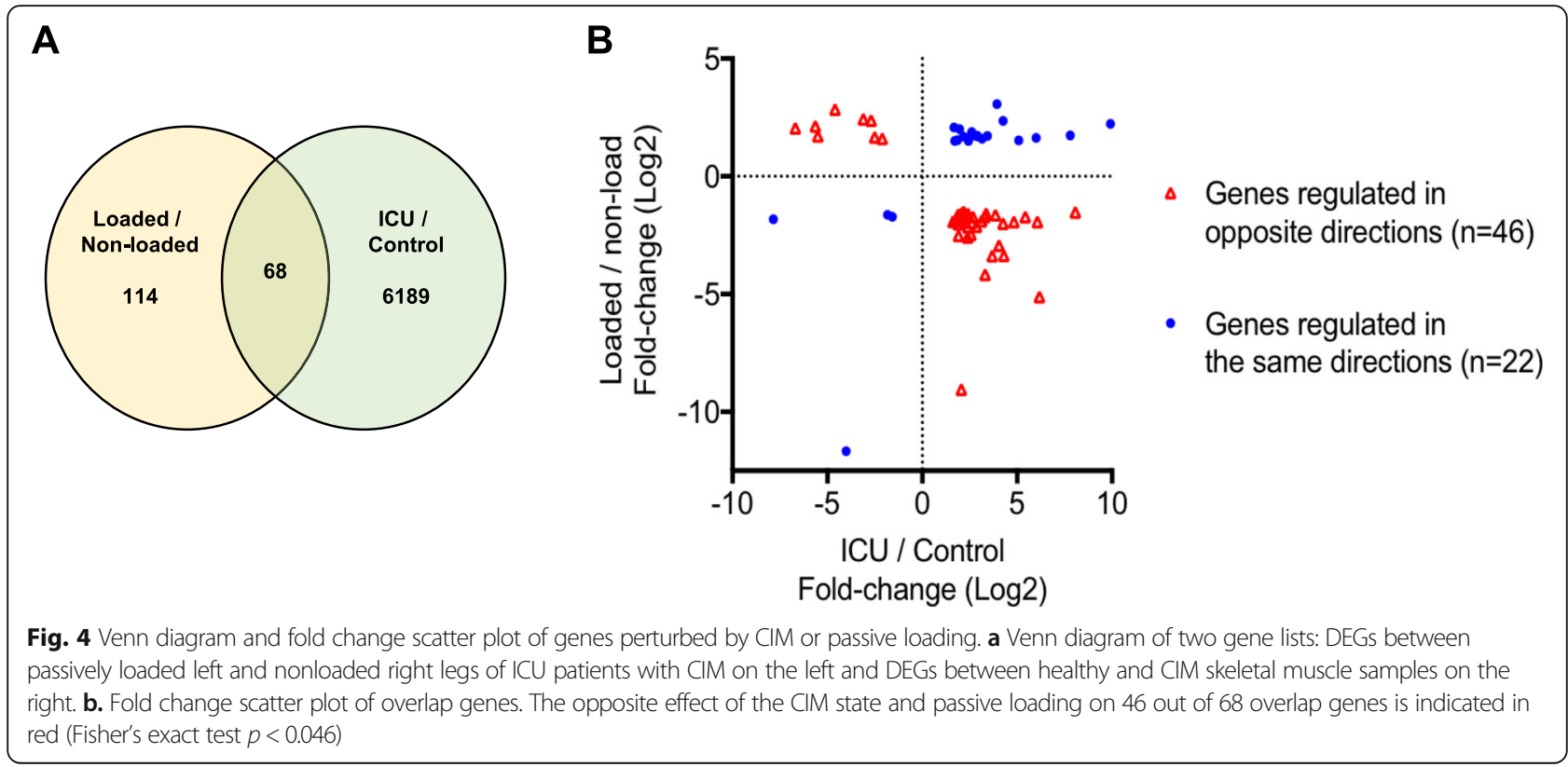

\section{Discussion}

CIM is a potentially lethal condition, affecting more than $30 \%$ of the ICU population and up to $100 \%$ in certain subgroups [5]. However, the gene expression and molecular pathways influencing skeletal muscle wasting and weakness in ICU patients with CIM are incompletely known. To our knowledge, this is the first time that RNA-seq was used to elucidate the skeletal muscle transcriptome profiling and upstream regulators of critically ill, immobilized, and mechanically ventilated ICU patients with CIM. Moreover, the effects of passive mechanical loading on the skeletal muscle transcriptome signature of patients with CIM have also been studied. Compared with traditional technologies to study gene expression, such as microarrays, high-throughput RNA-seq provides unprecedented sensitivity and reproducibility to discover DEGs while showing high accuracy for quantifying expression levels

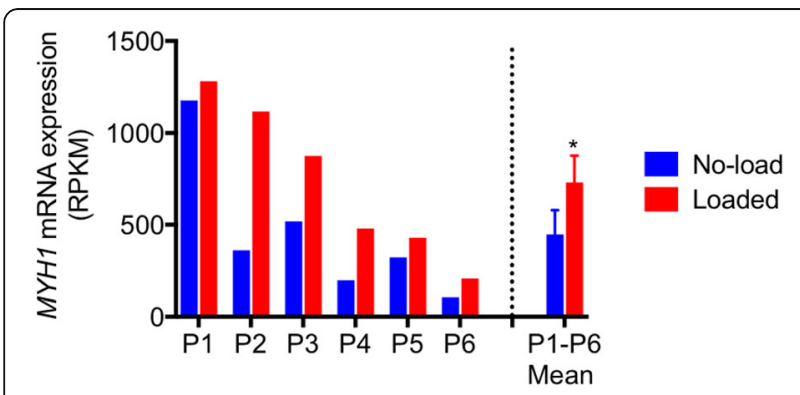

Fig. 5 MYH1 expression in muscle samples in different conditions. Significant increase of MYH1 expression in the ICU patient muscle samples and further increase in the loaded left compared to the nonloaded right legs and closely correlating with quantitative PCR and RNA spike-in control samples [26, 27].

Skeletal muscle adapts readily to environmental factors, nutritional interventions, loading conditions, and contractile activity by altering muscle fiber size, functional capacity, and metabolism. It has been demonstrated that mechanical silencing is a significant factor triggering the severe muscle atrophy and impaired function associated with CIM $[15,28]$, and the present results show a profound impact at the skeletal muscle transcriptional level in ICU patients with CIM. Out of 17,221 detected genes, 6257 (36\%) were affected in TA skeletal muscle from the patients with CIM. The expression of most of the genes was upregulated and revealed a high degree of similarity to the gene signatures available for patients with myopathy, polymyositis, sepsis, cancer, and immobilization, and the gene changes correlated best with gene signatures from patients with myopathy, immobilization, sepsis, polymyositis, and cancer. Ingenuity upstream regulator analysis revealed that genes regulated by MYOD1, p38 MAPK, and dexamethasone treatment are affected in the ICU patients with CIM. These molecules have established roles in skeletal muscle development and regeneration, as well as in response to cellular stress. MYOD1 is a transcription factor that regulates muscle cell differentiation and skeletal muscle regeneration and plasticity in response and hypertrophic or atrophic conditions $[21,22,29,30]$. P38 MAPK is involved in skeletal muscle differentiation and development and in cast-immobilized atrophy via MuRF1 [23, 31]. Dexamethasone is a glucocorticoid that can induce skeletal muscle atrophy and myosin loss associated with upregulation of MuRF1 and atrogin-1 and downregulation of HDAC6 [32, 33]. 
The three proteolytic systems (UPS, ALS, and the calcium-dependent calpains) were activated in CIM. In addition to the upregulation (although not statistically significant) of MURF1 and ATROGIN-1, two newly identified and critical E3 ligases that control muscle size, TRIM32 and FBXO30/MUSA1, were upregulated in response to muscle atrophy associated with CIM. TRIM32 binds to myosin and ubiquitinates desmin, as well as thin filament (actin, tropomyosin, troponins) and Z-band ( $\alpha$-actinin) proteins [34]. TRIM32 expression is not upregulated under all forms of atrophy, and its role in the regulation of muscle mass is still not clear [35]. Moreover, TRIM32 knockout mice develop atrophy and show impaired muscle regrowth after atrophy [36]. FBXO30/MUSA1 is upregulated by denervation-induced atrophy, and the knockdown of MUSA1 reduces muscle atrophy after denervation [37]. FBXO40 is upregulated during muscle differentiation, and FBXO40 knockout mice show increased muscle size [38]. FBXO40 expression was downregulated in patients with CIM. This result is reminiscent of findings in dystrophic muscles of limb-girdle muscular dystrophy (LGMD) patients and starvation-related muscle atrophy, but it contrasts with an upregulation in response to denervation-induced muscle atrophy [39].

Autophagy can be activated by starvation, exercise, critical illness, and a variety of stress signals [20]. Autophagy may contribute to muscle protein degradation by increased breakdown of damaged proteins/organelles or may protect myofiber integrity and muscle tissue homeostasis by clearing abnormal organelles and by sequestering cytotoxic products that result from myofibrillar protein degradation [40]. Recently, a considerable number of studies have confirmed a protective role of autophagy against various types of organ failure in critically ill animal models [41]. Several cathepsins, $M A P 1 L C 3 B$, which has an essential role in the formation of autophagosomes, and p62/SQSTM1, which mediates the breakdown of protein aggregates by binding to LC3 and polyubiquitinated proteins [42], had increased expression in our study. In addition, NAF1, a Bcl-2 associated autophagy regulator that is essential for the maintenance of skeletal muscle mass [43], was also upregulated. This indicates that activation of ALS plays an important role in patients with CIM, although its detrimental vs. protective roles need to be further studied.

The calpain system, which disassembles myofibrillar proteins from the sarcomere to be ubiquitinated and degraded, is activated in the ICU patients with CIM. The expression of the muscle-specific isoform CAPN3 decreased, in accordance with findings in animal models of CIM [12] and denervation-induced atrophy and regeneration [44]. However, CAPN3 is primarily involved in sarcomere remodeling and does not contribute to the increased protein degradation.
Altered chaperone expression has been reported in a porcine model of critical illness [45]. HSPB6 ( $\alpha$-crystallin-related) expression was downregulated in CIM patients, in accordance with our results [12]. HSPB6 plays several roles in skeletal muscle contractile machinery and is associated with troponin complexes in the protection from muscle atrophy, ischemia, hypertensive stress, and metabolic dysfunction [46]. UNC-45 is a critical chaperone for myosin folding, stability, and function and protects myosin from stress [47]. In the current study, UNC-45B expression was upregulated. Overexpression of UNC-45 in muscle cells results in increased myosin degradation and reduced or disorganized myofibrils [48]. Moreover, loss or overexpression of UNC-45 leads to defective myofibril organization in the skeletal muscle of zebrafish embryos [49]. UNC-45 binds to HSP90, acting as a cochaperone in myosin folding and protection against stress [47]. HSP9O expression was upregulated in parallel with $U N C-45 B$ in the studied patients with CIM. Therefore, the higher expression of both HSP90 and UNC45 results in disassembly of the myosin filament. HSP70 is a key chaperone family involved in the maintenance of muscle fiber integrity and regulation of muscle regeneration [50]. HSP70 expression was downregulated in ICU patients with CIM, contrary to rodent models of CIM [12]. Conversely, HSP70 expression is reduced during muscle inactivity and aging, leading to muscle atrophy and impaired function [50].

Apoptosis is implicated in the development of muscle atrophy induced by several conditions, i.e., disuse or aging, and is needed in the maintenance of skeletal muscle homeostasis [51, 52]. Apoptosis is orchestrated by caspases and activated by cell cycle regulators, particularly those that induced cell death, and proapoptotic genes such as PDCD6, AATF, ACIN1, AIFM1, APAF1, $M K R N 1$, and $B A X$, which were upregulated in the ICU patients with CIM; additionally, the caspases CASP4 and CASP6 and several genes related to the p53 pathway were activated. Activation of apoptosis at the mRNA level and increased myonuclear apoptosis have been previously reported in an experimental ICU model [12] in accordance with the results obtained from the ICU patients diagnosed with CIM.

Sarcomeric protein gene expression was significantly affected by CIM. The downregulation of $\mathrm{MyHC}$ isoforms and actin at the transcriptional level, despite decreased myosin to actin ratios at the protein level, has been previously reported during the acute phase of CIM [3]. This indicates that protein synthesis is affected. The differences between gene and protein expression might be due to posttranscriptional regulation, protein degradation, or protein turnover rate, as actin has a twofold longer protein turnover rate than myosin $[3,53]$. We have previously seen an upregulation of $M Y B P H$ in a rat 
model of CIM [12] and in ICU patients with CIM during recovery [3], suggesting a role of $M Y B P H$ in the maintenance and reassembly of the thick filament structure in patients with CIM [3].

The muscle protein translational machinery was activated in accordance with our previous observations in an experimental ICU model [12, 28]. In this context, it is interesting to note that stimulation of muscle protein synthesis signaling pathways has also been reported in critically ill ICU patients [54], despite marked muscle wasting, suggesting that protein degradation was exceeding the synthesis rate. The increased protein synthesis has been suggested to be related to an increased availability of amino acids from the increased proteolysis and/or an attempt to counteract muscle mass loss [54]. Moreover, the activation of several genes that play a critical role in skeletal muscle development and regeneration, such as myogenic factors (MYOG, MYF6) [55]; metalloproteinase $M M P 14$ [56]; the transcription factor SOX6, which also regulates muscle fiber type differentiation $[57,58]$; and $D M P K$, whose reduction also contributes to muscle wasting in muscular dystrophies [59], together with the transcriptional upregulation of genes that participate in the translational machinery, may indicate compensatory mechanisms to reduce the excessive sarcomeric degradation and muscle wasting seen under ICU conditions.

Surprisingly, passive mechanical loading for $10 \mathrm{~h}$ a day for $9 \pm 1$ days only marginally affected the skeletal muscle gene signature of another group of CIM patients. Single muscle fiber contractile measurements from the same samples were previously shown to increase specific force by $35 \%$ in response to passive loading [15]. These results could indicate that the improvement in specific muscle fiber force occurs primarily at the post-transcriptional level, but mass-spectrometry analyses of myosin posttranslational modifications (PTMs) did not show any significant differences between the loaded and the unloaded leg [15]. Other PTMs in thin filament proteins may be affected by the loading condition, with important consequences for the regulation of muscle contraction. In addition, it is important to emphasize that the present gene expression data reflect the average gene changes in the skeletal muscle tissue, whereas the specific force measurements were obtained from single fibers with sufficient integrity to undergo contractile measurements. Interestingly, the expression of MyHC-IIx/d (MYH1) increased $500 \%$ in the CIM state (Additional file 2) and further increased $64 \%$ in the loaded leg at the transcriptional level (Fig. 5 and Additional file 3), but these increases could not counteract the loss of myosin and muscle mass [15]. There is a slow turnover of myosin in skeletal muscle, and one cannot exclude that the initial preferential myosin loss targets a pool of newly synthesized myosin or myosin destined for degradation (that is, myosin not involved in force generation). In previous experimental studies, we have shown that activation of different protein synthesis and degradation pathways in response to mechanical ventilation and immobilization follow a strict temporal pattern [12, 28, 60]. For logistic reasons, all gene expression measurements in this study were conducted at one late time point during the unilateral mechanical loading in immobilized and mechanically ventilated ICU patients. Therefore, it cannot be excluded that early activation of mechanosensitive pathways that preceded the time of muscle biopsy collection have gone undetected in the current study. In an attempt to improve our understanding of the temporal gene expression pattern in ICU patients developing CIM, we are presently following a group of ICU patients with six repeated muscle biopsies during 12 days of mechanical ventilation and immobilization. Another potential explanation for the small difference in gene expression between loaded and unloaded leg is the crossover effects on gene expression via circulating factors released from the loaded muscles, such as myokines or exosomes. Based on our previous experimental work, we do no not find any experimental evidence of a crossover effect by unilateral mechanical loading [19, 61]. However, recent experimental findings from our group show a strong crossover effect in response to unilateral direct muscle electrical stimulation, presumably generated via calcium-stimulated exosome release mediating muscleto-muscle communication (in preparation).

\section{Conclusions}

Mechanical silencing in critically ill ICU patients who developed CIM is associated with profound changes in skeletal muscle gene expression. RNA-seq analysis revealed that the marked muscle atrophy and weakness seen in ICU patients with CIM were associated with altered expression of genes involved in muscle contraction and system process, newly identified E3 ligases, autophagy and calpain systems, apoptosis, and chaperone expression at the transcriptional level. Ingenuity upstream regulator analysis additionally revealed that the CIM signature is reminiscent of those detected by activation of MYOD1, p38 MAPK, or treatment with dexamethasone. However, mechanical loading only marginally affected the skeletal muscle transcriptome profiling of ICU patients diagnosed with CIM.

\section{Additional files}

Additional file 1: RNA quality number (RQN). (XLSX 13 kb)

Additional file 2: 6257genes_withFDR. (TXT $284 \mathrm{~kb}$ )

Additional file 3: 182genes_withFDR. (TXT 8 kb) 
Additional file 4: Of these 68 genes, 46 were regulated in the opposite direction, and 22 genes were regulated in the same direction. (XLSX $14 \mathrm{~kb}$ )

\section{Abbreviations}

ALS: Autophagy-lysosome system; CIM: Critical illness myopathy; DEGs: Differentially expressed genes; GO: Gene Ontology; HSP: Heat shock protein; ICU: Intensive care unit; MyHC: Myosin heavy chain; PCA: Principal component analysis; PTMs: Post-translational modifications; RNA-seq: RNA sequencing; RPKM: Reads per kilobase per million; TA: Tibialis anterior: UBEs: Ubiquitin-conjugating enzymes; UPS: Ubiquitin proteasome system

\section{Acknowledgements}

We are grateful to Ms. Yvette Hedström for excellent technical assistance.

\section{Funding}

The study was supported by grants from the Erling Persson Family Foundation, the Swedish Research Council (8651), Stockholm City Council (Alf 20150423, 20170133), and Karolinska Institutet to L.L.

\section{Availability of data and materials}

Raw sequencing reads generated from this study are deposited at the NCB sequence read archive (SRA) under accession PRJNA491748.

\section{Authors' contributions}

WF, LL, and JG designed the research. WF, MLD, YB, and JG analyzed the data. WF, MLD, HO, LL, and JG wrote the paper. All authors approved the final version of the manuscript.

\section{Ethics approval and consent to participate}

The study was approved by the Ethical Committee on Human Research at Uppsala University Hospital, Uppsala, Sweden.

\section{Consent for publication}

Authors declare no conflict of interest.

\section{Competing interests}

WF, $\mathrm{HO}$, and $\mathrm{YB}$ are employees and shareholders of Regeneron Pharmaceuticals. JG was an employee and shareholder of Regeneron Pharmaceuticals during the completion of the study.

\section{Publisher's Note}

Springer Nature remains neutral with regard to jurisdictional claims in published maps and institutional affiliations.

\section{Author details}

'Department of Physiology and Pharmacology, Karolinska Institutet Bioclinicum, J8:30, SE-171 77 Stockholm, Sweden. ${ }^{2}$ Regeneron Pharmaceuticals, Inc., Tarrytown, NY 10591, USA. ${ }^{3}$ Department of Clinical Neuroscience, Karolinska Institutet and Karolinska Hospital, Stockholm, Sweden.

\section{Received: 26 September 2018 Accepted: 31 March 2019} Published online: 16 April 2019

\section{References}

1. Ingber DE. Control of capillary growth and differentiation by extracellular matrix. Use of a tensegrity (tensional integrity) mechanism for signal processing. Chest. 1991;99(3 Suppl):34S-40S.

2. Raskin AM, Hoshijima M, Swanson E, McCulloch AD, Omens JH. Hypertrophic gene expression induced by chronic stretch of excised mouse heart muscle. Mol Cell Biomech. 2009:6(3):145-59.

3. Norman H, Zackrisson H, Hedstrom Y, Andersson P, Nordquist J, Eriksson LI, Libelius R, Larsson L. Myofibrillar protein and gene expression in acute quadriplegic myopathy. J Neurol Sci. 2009;285(1-2):28-38.

4. Larsson L, Li XP, Edstrom L, Eriksson LI, Zackrisson H, Argentini C, Schiaffino S. Acute quadriplegia and loss of muscle myosin in patients treated with nondepolarizing neuromuscular blocking agents and corticosteroids: mechanisms at the cellular and molecular levels. Crit Care Med. 2000;28(1):34-45.
5. Friedrich O, Diermeier $\mathrm{S}$, Larsson L. Weak by the machines: muscle motor protein dysfunction - a side effect of intensive care unit treatment. Acta Physiol (Oxf). 2018;222(1).

6. Rudis MI, Guslits BJ, Peterson EL, Hathaway SJ, Angus E, Beis S, Zarowitz BJ. Economic impact of prolonged motor weakness complicating neuromuscular blockade in the intensive care unit. Crit Care Med. 1996; 24(10):1749-56

7. Seneff MG, Wagner D, Thompson D, Honeycutt C, Silver MR. The impact of long-term acute-care facilities on the outcome and cost of care for patients undergoing prolonged mechanical ventilation. Crit Care Med. 2000:28(2):342-50.

8. Lacomis D. Electrophysiology of neuromuscular disorders in critical illness. Muscle Nerve. 2013:47(3):452-63.

9. Larsson L, Friedrich O. Critical illness myopathy (CIM) and ventilator-induced diaphragm muscle dysfunction (VIDD): acquired myopathies affecting contractile proteins. Compr Physiol. 2016;7(1):105-12.

10. Shepherd S, Batra A, Lerner DP. Review of critical illness myopathy and neuropathy. Neurohospitalist. 2017;7(1):41-8.

11. Langhans C, Weber-Carstens S, Schmidt F, Hamati J, Kny M, Zhu X, Wollersheim T, Koch S, Krebs M, Schulz H, et al. Inflammation-induced acute phase response in skeletal muscle and critical illness myopathy. PLoS One. 2014;9(3):e92048.

12. Llano-Diez M, Gustafson AM, Olsson C, Goransson H, Larsson L. Muscle wasting and the temporal gene expression pattern in a novel rat intensive care unit model. BMC Genomics. 2011;12:602.

13. Banduseela VC, Ochala J, Chen YW, Goransson H, Norman H, Radell P, Eriksson LI, Hoffman EP, Larsson L. Gene expression and muscle fiber function in a porcine ICU model. Physiol Genomics. 2009;39(3):141-59.

14. Griffiths RD, Palmer TE, Helliwell T, MacLennan P, MacMillan RR. Effect of passive stretching on the wasting of muscle in the critically ill. Nutrition. 1995;11(5):428-32.

15. Llano-Diez M, Renaud G, Andersson M, Marrero HG, Cacciani N, Engquist $H$, Corpeño R, Artemenko K, Bergquist J, Larsson L. Mechanisms underlying ICU muscle wasting and effects of passive mechanical loading. Crit Care. 2012; 16(5):R209.

16. Renaud G, Llano-Diez M, Ravara B, Gorza L, Feng HZ, Jin JP, Cacciani N, Gustafson AM, Ochala J, Corpeno R, et al. Sparing of muscle mass and function by passive loading in an experimental intensive care unit model. J Physiol. 2013;591(Pt 5):1385-402.

17. Stibler H, Edstrom L, Ahlbeck K, Remahl S, Ansved T. Electrophoretic determination of the myosin/actin ratio in the diagnosis of critical illness myopathy. Intensive Care Med. 2003:29(9):1515-27.

18. Frontera WR, Larsson L. Contractile studies of single human skeletal muscle fibers: a comparison of different muscles, permeabilization procedures, and storage techniques. Muscle Nerve. 1997;20(8):948-52.

19. Corpeno Kalamgi R, Salah H, Gastaldello S, Martinez-Redondo V, Ruas JL, Fury W, Bai Y, Gromada J, Sartori R, Guttridge DC, et al. Mechano-signalling pathways in an experimental intensive critical illness myopathy model. J Physiol. 2016;594(15):4371-88.

20. Sandri M. Protein breakdown in muscle wasting: role of autophagy-lysosome and ubiquitin-proteasome. Int J Biochem Cell Biol. 2013:45(10):2121-9.

21. Rudnicki MA, Schnegelsberg PN, Stead RH, Braun T, Arnold HH, Jaenisch R. MyoD or Myf-5 is required for the formation of skeletal muscle. Cell. 1993; 75(7):1351-9.

22. Ishido M, Kami K, Masuhara M. Localization of MyoD, myogenin and cell cycle regulatory factors in hypertrophying rat skeletal muscles. Acta Physiol Scand. 2004;180(3):281-9.

23. Keren A, Tamir Y, Bengal E. The p38 MAPK signaling pathway: a major regulator of skeletal muscle development. Mol Cell Endocrinol. 2006;252(12):224-30.

24. Qin J, Du R, Yang YQ, Zhang HQ, Li Q, Liu L, Guan H, Hou J, An XR. Dexamethasone-induced skeletal muscle atrophy was associated with upregulation of myostatin promoter activity. Res Vet Sci. 2013;94(1):84-9

25. Renaud G, Llano-Diez M, Ravara B, Gorza L, Feng HZ, Jin JP, Cacciani N, Gustafson AM, Ochala J, Corpeno R, et al. Sparing of muscle mass and function by passive loading in an experimental intensive care unit model. J Physiol. 2013;591(Pt 5:1385-402.

26. Wang Z, Gerstein M, Snyder M. RNA-Seq: a revolutionary tool for transcriptomics. Nat Rev Genet. 2009;10(1):57-63.

27. Nagalakshmi U, Waern K, Snyder M. RNA-Seq: a method for comprehensive transcriptome analysis. Curr Protoc Mol Biol. 2010; Chapter 4:Unit 4.11.11-13. 
28. Ochala J, Gustafson AM, Diez ML, Renaud G, Li M, Aare S, Qaisar R, Banduseela VC, Hedstrom Y, Tang X, et al. Preferential skeletal muscle myosin loss in response to mechanical silencing in a novel rat intensive care unit model: underlying mechanisms. J Physiol. 2011;589(Pt 8:2007-26.

29. Davis $R L$, Weintraub $H$, Lassar $A B$. Expression of a single transfected cDNA converts fibroblasts to myoblasts. Cell. 1987;51(6):987-1000.

30. Ishido M, Kami K, Masuhara M. In vivo expression patterns of MyoD, p21, and $\mathrm{Rb}$ proteins in myonuclei and satellite cells of denervated rat skeletal muscle. Am J Physiol Cell Physiol. 2004;287(2):C484-93.

31. Kim J, Won KJ, Lee HM, Hwang BY, Bae YM, Choi WS, Song H, Lim KW, Lee CK, Kim B. p38 MAPK participates in muscle-specific RING finger 1-mediated atrophy in cast-immobilized rat gastrocnemius muscle. Korean J Physiol Pharmacol. 2009:13(6):491-6.

32. Alamdari N, Smith IJ, Aversa Z, Hasselgren PO. Sepsis and glucocorticoids upregulate p300 and downregulate HDAC6 expression and activity in skeletal muscle. Am J Physiol Regul Integr Comp Physiol. 2010;299(2):R509-20

33. Clarke BA, Drujan D, Willis MS, Murphy LO, Corpina RA, Burova E, Rakhilin SV, Stitt TN, Patterson C, Latres E, et al. The E3 ligase MuRF1 degrades myosin heavy chain protein in dexamethasone-treated skeletal muscle. Cell Metab. 2007:6(5):376-85.

34. Cohen S, Zhai B, Gygi SP, Goldberg AL. Ubiquitylation by Trim32 causes coupled loss of desmin, Z-bands, and thin filaments in muscle atrophy. J Cell Biol. 2012;198(4):575-89.

35. Bodine SC, Baehr LM. Skeletal muscle atrophy and the E3 ubiquitin ligases MuRF1 and MAFbx/atrogin-1. Am J Physiol Endocrinol Metab. 2014;307(6): E469-84.

36. Kudryashova E, Kramerova I, Spencer MJ. Satellite cell senescence underlies myopathy in a mouse model of limb-girdle muscular dystrophy $2 \mathrm{H}$. J Clin Invest. 2012;122(5):1764-76.

37. Sartori R, Schirwis E, Blaauw B, Bortolanza S, Zhao J, Enzo E, Stantzou A, Mouisel E, Toniolo L, Ferry A, et al. BMP signaling controls muscle mass. Nat Genet. 2013;45(11):1309-18.

38. Shi J, Luo L, Eash J, Ibebunjo C, Glass DJ. The SCF-Fbxo40 complex induces IRS1 ubiquitination in skeletal muscle, limiting IGF1 signaling. Dev Cell. 2011; 21(5):835-47.

39. Ye J, Zhang Y, Xu J, Zhang Q, Zhu D. FBXO40, a gene encoding a novel muscle-specific F-box protein, is upregulated in denervation-related muscle atrophy. Gene. 2007:404(1-2):53-60.

40. Bonaldo P, Sandri M. Cellular and molecular mechanisms of muscle atrophy. Dis Model Mech. 2013;6(1):25-39.

41. Gunst J. Recovery from critical illness-induced organ failure: the role of autophagy. Crit Care. 2017;21(1):209.

42. Sandri M. Autophagy in skeletal muscle. FEBS Lett. 2010;584(7):1411-6.

43. Chang NC, Nguyen M, Bourdon J, Risse PA, Martin J, Danialou G, Rizzuto R, Petrof BJ, Shore GC. BCl-2-associated autophagy regulator Naf-1 required for maintenance of skeletal muscle. Hum Mol Genet. 2012;21(10):2277-87.

44. Stockholm D, Herasse M, Marchand S, Praud C, Roudaut C, Richard I, Sebille A, Beckmann JS. Calpain 3 mRNA expression in mice after denervation and during muscle regeneration. Am J Physiol Cell Physiol. 2001;280(6):C1561-9.

45. Banduseela VC, Chen YW, Kultima HG, Norman HS, Aare S, Radell P, Eriksson LI, Hoffman EP, Larsson L. Impaired autophagy, chaperone expression, and protein synthesis in response to critical illness interventions in porcine skeletal muscle. Physiol Genomics. 2013;45(12):477-86.

46. Dreiza CM, Komalavilas P, Furnish EJ, Flynn CR, Sheller MR, Smoke CC, Lopes $\mathrm{LB}$, Brophy CM. The small heat shock protein, HSPB6, in muscle function and disease. Cell Stress Chaperones. 2010;15(1):1-11.

47. Lee CF, Melkani GC, Bernstein SI. The UNC-45 myosin chaperone: from worms to flies to vertebrates. Int Rev Cell Mol Biol. 2014;313:103-44.

48. Landsverk ML, Li S, Hutagalung AH, Najafov A, Hoppe T, Barral JM, Epstein HF. The UNC-45 chaperone mediates sarcomere assembly through myosin degradation in Caenorhabditis elegans. J Cell Biol. 2007;177(2):205-10.

49. Bernick EP, Zhang PJ, Du S. Knockdown and overexpression of Unc-45b result in defective myofibril organization in skeletal muscles of zebrafish embryos. BMC Cell Biol. 2010;11:70.

50. Senf SM. Skeletal muscle heat shock protein 70: diverse functions and therapeutic potential for wasting disorders. Front Physiol. 2013;4:330.

51. Dirks-Naylor AJ, Lennon-Edwards S. Cellular and molecular mechanisms of apoptosis in age-related muscle atrophy. Curr Aging Sci. 2011;4(3):269-78.

52. Dupont-Versteegden EE. Apoptosis in skeletal muscle and its relevance to atrophy. World J Gastroenterol. 2006;12(46):7463-6.
53. Martin AF. Turnover of cardiac troponin subunits. Kinetic evidence for a precursor pool of troponin-I. J Biol Chem. 1981;256(2):964-8.

54. Jespersen JG, Nedergaard A, Reitelseder S, Mikkelsen UR, Dideriksen K, Agergaard J, Kreiner F, Pott FC, Schjerling P, Kjaer M. Activated protein synthesis and suppressed protein breakdown signaling in skeletal muscle of critically ill patients. PLoS One. 2011;6(3):e18090.

55. Zammit PS. Function of the myogenic regulatory factors Myf5, MyoD, Myogenin and MRF4 in skeletal muscle, satellite cells and regenerative myogenesis. Semin Cell Dev Biol. 2017;72:19-32.

56. Snyman C, Niesler CU. MMP-14 in skeletal muscle repair. J Muscle Res Cell Motil. 2015;36(3):215-25

57. Hagiwara N, Ma B, Ly A. Slow and fast fiber isoform gene expression is systematically altered in skeletal muscle of the Sox6 mutant, p100H. Dev Dyn. 2005;234(2):301-11.

58. Hagiwara N, Yeh M, Liu A. Sox6 is required for normal fiber type differentiation of fetal skeletal muscle in mice. Dev Dyn. 2007;236(8):2062-76.

59. Harmon EB, Harmon ML, Larsen TD, Yang J, Glasford JW, Perryman MB. Myotonic dystrophy protein kinase is critical for nuclear envelope integrity. J Biol Chem. 2011;286(46):40296-306.

60. Corpeno R, Dworkin B, Cacciani N, Salah H, Bergman HM, Ravara B, Vitadello M, Gorza L, Gustafson AM, Hedstrom Y, et al. Time course analysis of mechanical ventilation-induced diaphragm contractile muscle dysfunction in the rat. J Physiol-London. 2014;592(17):3859-80,

61. Renaud G, Llano-Diez M, Ravara B, Gorza L, Feng HZ, Jin JP, Cacciani N, Gustafson AM, Ochala J, Corpeno R, et al. Sparing of muscle mass and function by passive loading in an experimental intensive care unit model. J Physiol. 2013;591(5):1385-402.

\section{Ready to submit your research? Choose BMC and benefit from:}

- fast, convenient online submission

- thorough peer review by experienced researchers in your field

- rapid publication on acceptance

- support for research data, including large and complex data types

- gold Open Access which fosters wider collaboration and increased citations

- maximum visibility for your research: over $100 \mathrm{M}$ website views per year

At BMC, research is always in progress.

Learn more biomedcentral.com/submissions 